\title{
The contributions of smell and taste to overall intensity: A model
}

\author{
DAVID E. HORNUNG and MELVIN P. ENNS \\ St. Lawrence University, Canton, New York
}

\begin{abstract}
A model is proposed to describe mathematically the integration of olfaction and gustation in producing the sensation of overall intensity or flavor. The basis of this additive model is the premise that the sensation of overall intensity is composed of the summation of the estimates of the intensities of smell and taste. However, since this summation has been consistently shown to be greater than the estimates of the intensity of flavor, the additive model is modified such that the psychophysical functions describing the magnitude estimates of smell and taste are "reduced" when used to predict the overall intensity or flavor. The model's predictive capacity is demonstrated by the results of a test using the Two-Module Delivery System (Hornung \& Enns, 1984) to present the odorant ethyl butyrate and the tastant sucrose. Using all combinations of distilled water and three concentrations of the odorant combined with distilled water and three concentrations of the tastant, subjects used the method of absolute magnitude estimation to scale the intensities of smell, taste, and flavor. Estimates of overall intensity (flavor) were predicted accurately from the intensity ratings given to smell and taste.
\end{abstract}

Although it is generally agreed that olfaction and gustation add together in some way to produce the sensation of flavor, the exact definition of this additivity has been less certain. The results of one set of experiments seem to indicate that the additivity is essentially $100 \%$, whereas other results suggest that the estimate of overall intensity is significantly less than the sum of the olfactory and gustatory components. In part, this apparent conflict occurs because of the difference in judgmental tasks given to the subjects. The present paper will determine the extent to which judgments of overall intensity can be predicted from focused judgments of taste alone and smell alone. From these predictions comes a mathematical model that describes the contributions of taste and smell in determining the overall intensity of a flavor stimulus.

The data supporting the suggestion of complete (100\%) additivity have come primarily from the pioneering work of Murphy and Cain (1980) and Murphy, Cain, and Bartoshuk (1977). Murphy et al. used the odorant ethyl butyrate and the tastant sodium saccharin and found that the estimated overall intensity of the flavor mixture was $93 \%$ of the sum of the estimates of the overall intensities of the smell and taste. Murphy and Cain found $85 \%$ and $90 \%$ additivity for the odorant citral with the tastants sucrose and sodium chloride. Even though these results did not show $100 \%$ additivity, correcting the estimates of the taste and smell intensities by subtracting the taste and smell intensities reported for distilled water (subtraction of false positives) yielded a picture of complete additivity (Murphy \& Cain, 1980).

This work was supported by a grant from the General Foods Corporation. D. E. Hornung is with the Biology Department and M. P. Enns is with the Psychology Department, St. Lawrence University, Canton, NY 13617.
In both of the studies mentioned above, estimates of the intensity of the odorant, as well as the olfactory component of the flavor mixture, came through the mouth. That is, subjects placed the stimuli in their mouths and then judged the overall intensity of the smell, taste, or flavor. With this method of stimulus presentation, the odorant(s) could reach the appropriately tuned receptors only by moving from the oral cavity to the headspace above the olfactory receptors (a condition referred to as "retronasal olfaction" by Burdach, Kroeze, \& Koster, 1984). In these experiments, the overall intensity of the flavor mixture was compared to the sum of the overall intensity of the odorant and the overall intensity of the tastant.

The results supporting the suggestion that the estimate of overall intensity is significantly less than the sum of the olfactory and gustatory components have come from studies in which the odorant molecules were directed to the olfactory receptors as a result of a sniff ("nasal olfaction," Burdach et al., 1984). Also, in these studies, the overall intensity of the flavor was compared to the estimates of the intensity of the odor and taste, not the overall intensity of the odorant and tastant. Enns and Hornung (1985) used a solution of almond extract as both the odorant and tastant and found only a $67 \%$ additivity. Likewise, Garcia-Medina (1981) reported an $80 \%$ additivity with solutions of acetic acid and a 57\% additivity with solutions of coffee. Applying a correction for the estimates of the intensity of the smell and taste of distilled water would not produce a picture of complete additivity for either the data of Enns and Hornung or that of GarciaMedina.

Enns and Hornung's (1985) results were obtained using the Two-Module Delivery System (Hornung \& Enns, 1984) to present the stimulus. This delivery system has 
the advantage over the open-cup delivery system of permitting the concentration of a stimulus delivered to the nose to be varied independently of the concentration delivered to the mouth. However, with the Two-Module Delivery System the relative timing of the olfactory and gustatory stimuli must be considered. That is, because the system requires that a subject smell and then taste the flavor solution, the smell and taste occur sequentially.

It must be recognized that the open-cup delivery system also results in a sequential stimulation of the nose and mouth. That is, after a stimulus is placed in the mouth, odorant molecules must diffuse through the nasopharynx to reach the olfactory receptors, whereas the gustatory molecules are applied directly to the taste receptor area. However, the difference between the onsets of the olfactory and gustatory components of a flavor stimulus is in all likelihood greater with the Two-Module Delivery System than with the open-cup delivery system. Therefore, before we attempted to reconcile all the above data it was necessary to determine how much of the suppression observed by Enns and Hornung (1985) was due to the method of stimulus presentation. Thus, in Experiment 1 the scaling techniques employed by Enns and Hornung (1985) were used to determine the intensity of oral olfactory, gustatory, and flavor stimuli presented to the subjects with an open-cup delivery system.

\section{EXPERIMENT 1}

\section{Method}

The stimuli were solutions of ethyl butyrate (Eastman Organic Chemicals Co., Rochester, NY) and sucrose (Sunny Square brand, purchased from a local supermarket) prepared in distilled water. These stimuli were chosen because each had been used in previous experiments to explore the components of flavor (Gillan, 1983; Murphy \& Cain, 1980; Murphy et al., 1977). Three stimulus mixtures were prepared: low intensity $(0.01 \%$ ethyl butyrate with $5.0 \%$ sucrose), moderate intensity $(0.04 \%$ ethyl butyrate with $10.0 \%$ sucrose, and high intensity ( $0.16 \%$ ethyl butyrate with $20 \%$ sucrose). (All ethyl butyrate concentrations were $v / v$; all sucrose concentrations were $w / v$.) The stimuli were delivered via standard medicine cups, with $10 \mathrm{ml}$ available for each trial. Ten undergraduate college students served as subjects.

To acquaint the subjects with the method of absolute magnitude estimation (AME), the experimenters had each subject estimate the length of seven lines, using a technique reported in detail elsewhere (Enns \& Hornung, 1985; Zwislocki \& Goodman, 1980). After placing each mixture in their mouths, the subjects were asked to use the AME method to estimate the intensity of the odor. On separate trials, the subjects rated the intensity of the taste of each solution. Finally, the subjects were asked to estimate the overall intensity of the three mixtures. They were instructed to place the solution in their mouths and then answer the question "What is the overall intensity of the solution?" Overall intensity was defined as "some combination of the smell and taste." Each of the odorant/tastant combinations was presented three times for a total of nine trials.

\section{Results}

The arithmetic mean of the three estimates of the odor, taste, and overall intensity of each concentration was calculated and used in the data analysis.
On the average, estimates of the overall intensity of the ethyl butyrate/sucrose mixtures were approximately $60 \%$ of the sum of the estimates of the intensities of the smell and taste of the ethyl butyrate/sucrose mixtures (Table 1). Thus, even when the entire flavor solution was presented in the mouth via an open-cup delivery system, the estimates of overall intensity were substantially lower than the sum of the estimates of olfaction and taste. Therefore, the results obtained with the Two-Module Delivery System do not appear to be unique to this delivery system. It is with this background that we propose the following model as an attempt to describe the relationship between olfaction, taste, and overall intensity.

\section{THE MODEL}

Since all of the experimental data suggest that the sensation of overall intensity is composed of the summation of the estimates of the intensities of smell and taste, the general form of the relationship between smell, taste, and overall intensity should be:

$$
\Psi_{\text {Overall Intensity }} \propto \Psi_{\text {Smell }}+\Psi_{\text {Taste }} .
$$

However, we propose that this summation is greater than the estimates of overall intensity. We suggest that this lack of complete additivity is accomplished by the "reduction" of the psychophysical functions of smell and taste by constant factors $k_{s}$ and $k_{t}$. Thus the proportionality becomes:

$$
\Psi_{\text {Overall Intensity }}=k_{s}\left(\Psi_{\text {Smell }}\right)+k_{t}\left(\Psi_{\text {Taste }}\right) \text {. }
$$

Considering that the range of the percent additivity for all the stimulus pairs so far studied is between $57 \%$ and $90 \%$, it seems reasonable to assume that the values for $k_{s}$ and $k_{\mathrm{t}}$ are closer to 1 than to 0 , and that the values for the constants $k_{s}$ and $k_{t}$ are probably stimulus specific.

The use of correction factors in this model seems to be justified on the basis of previous studies in which the odorant was manipulated independently of the tastant. When all combinations of four concentrations of instant coffee (Hornung \& Enns, 1984) or almond extract (Enns \& Hornung, 1985) were used, estimates of overall intensity were shown to be composed of the sum of the arithmetic estimates of the tastant and odorant. When overall intensity was plotted against the concentration of either

Table 1

Mean Estimates of Overall Intensity Compared with the Summation of the Mean Estimates of the Intensity of Smell and Taste

\begin{tabular}{lccc}
\hline & \multicolumn{3}{c}{ Ethyl Butyrate and Sucrose } \\
\cline { 2 - 4 } & Low & Moderate & High \\
\hline Smell & 7.4 & 14.2 & 19.0 \\
Taste & 10.4 & 20.8 & 30.5 \\
Overall & & & \\
Intensity & 10.0 & 19.3 & 33.7 \\
Percent & $56.2 \%$ & $55.0 \%$ & $68.1 \%$ \\
\hline
\end{tabular}

Note-Percent $=[$ Overall Intensity $/($ Smell + Taste $)] \times 100$. 
the odorant or the tastant, parallel lines emerged. These parallel lines, without any statistical interaction, suggest that a taste number is being added to a smell number to produce the sensation of overall intensity (Anderson, 1970). Also, since the relation between the perceived overall intensity and the sum of the perceived smell and taste intensities did not change as the concentration of the odorant or tastant was changed, it was assumed that the olfactory and gustatory components were being suppressed in the mathematical manner described above.

The values of $k$ could be experimentally determined if overall intensity was judged when either perceived taste or smell was equal to zero. Then, if the above model is correct, the estimates of overall intensity would be less than the estimates of the intensity of the smell or taste alone. Furthermore, the $k_{s}$ would be the antilog of the difference between the log estimate of the intensity of the odorant and the log estimate of the overall intensity with no taste stimuli present. The same logic would apply for $k_{t}$ when the smell was equal to zero. In these situations, the percent suppression would reflect the $k$ term of either taste or smell. (The larger the percent suppression, the greater the difference between the ratings of overall intensity and the sum of the ratings for smell and taste. When smell and taste are equal to overall intensity, the percent suppression is equal to zero.)

Since nasal olfaction does not preclude retronasal olfaction, and vice versa (Enns \& Hornung, 1985), the relationship of retronasal olfaction to the olfactory term in Equation 2 needs to be considered. That is, whenever the presence of a retronasal smell can be documented, and this smell occurs along with a nasal smell sensation, the combination of these two smells could have a number of possible effects on Equation 2. Unfortunately, almost nothing is known about the way in which smell sensations from two locations influence the perception of odorants. Therefore, the olfactory term in Equation 2 is intended to reflect the psychophysical function of the total olfactory component of a flavor mixture. Exactly how nasal and retronasal smell add together to produce this total olfactory sensation needs to be determined experimentally (Burdach et al., 1984).

It is necessary to have data on a larger number of chemicals before we can determine the accuracy of Equation 2 in predicting the intensity of flavor from the intensities of the olfactory and gustatory components. In addition, flavor certainly involves stimulus parameters other than intensity of smell and taste (e.g., texture, color, and temperature). The final equation describing flavor will have to consider these and many other factors.

However, the proposed model seems to describe previously reported data. For example, Cometto-Muniz (1981) suggested that the perception of overall intensity reflected the more prominent feature of a taste/smell mixture. If, as the model suggests, taste and smell sensations add together to produce the intensity of flavor, and if one of these sensations is significantly larger than the other, then in one sense the overall intensity does reflect primarily the more prominent feature. Indeed, given the concentrations and stimuli used by Cometto-Muniz, the present model seems to predict the overall intensity data that was reported. Additionally, the present model apparently predicts the results when olfaction and gustation are approximately equal, a limitation of the explanation offered by Cometto-Muniz.

One of the assumptions of the present model is that the kind of judgment (overall intensity or modality-specific) has some influence on the perception of intensity. The model is conceived such that overall intensity of a taste/smell mixture can be predicted from the corrected intensities of taste and smell. These corrections represent a measure of the amount by which smell and taste are reduced when the subject estimates overall intensity. The product of $k_{s}$ and the intensity of the smell is thus equal to the overall intensity that one would assign to the smell when the taste was equal to zero. Likewise, the product of $k_{t}$ and the intensity of the taste is equal to the overall intensity that one would assign to the taste when smell was equal to zero.

Garcia-Medina (1981) proposed a vector summation model, as previously applied to odor mixtures (Berglund, Berglund, Lindvall, \& Svensson, 1973), to describe mathematically the lack of complete additivity that she observed. However, Enns and Hornung's (1985) results indicate that overall intensity does not equal taste when smell equals zero. Likewise, overall intensity does not equal smell when taste equals zero. Therefore, the vector model does not seem to be a likely explanation of the way in which perceived taste and smell interact to produce the sensation of flavor.

As an empirical test, we conducted two experiments so that the estimates of overall intensity of odorant/tastant mixtures predicted by the model could be compared to estimates actually given by the subjects. In these experiments, the odorant was again ethyl butyrate and the tastant was sucrose. Three concentrations $(0.01 \%, 0.04 \%$, and $0.16 \%$ ) of ethyl butyrate and three concentrations $(5.0 \%, 10.0 \%$, and $20.0 \%)$ of sucrose were prepared in distilled water. The subjects smelled and/or tasted all stimuli, which were delivered via the Two-Module Delivery System (Hornung \& Enns, 1984).

\section{EXPERIMENT 2}

\section{Method}

Twelve undergraduate college students, experimentally naive as to the psychophysical techniques of scaling smell and taste, served as subjects. They were asked to use the AME method to estimate first the lengths of lines and then the intensity of the odor of distilled water and the three concentrations of ethyl butyrate. The first solution given was always the middle concentration of the odorant solutions. Each of the remaining solutions was presented individually, with the order of presentation randomized. Each solution was presented three times for a total of 12 trials. The intensity of the odorant was determined following a sniff (Hornung \& Enns, 1984). The remainder of the procedure for determining the intensity of the odorants was identical to that described previously (Enns \& Hornung, 1985). Next, each subject was asked to use the AME method 
to estimate the intensity of the taste of distilled water and the three concentrations of sucrose. The methodology was the same as that described for the estimates of the smell of ethyl butyrate, except, of course, that subjects took the stimuli into their mouths and then estimated the intensity of taste.

Finally, the subjects were asked to estimate the overall intensity of all combinations of the odorants and tastants. They were instructed first to smell and then to taste the solution, and then to answer the question "What is the overall intensity of the solution?" Overall intensity was defined as "some combination of the smell and taste." Each of the odorant/tastant combinations was presented twice for a total of 32 trials.

\section{Results}

The psychophysical function (log-log coordinates) describing the scaling of the odor of ethyl butyrate was $m=0.23 ; Y=1.18 ; r=1.00$. The psychophysical function (log-log coordinates) describing the scaling of the taste of sucrose was $m=0.70 ; Y=0.10 ; r=1.00$.

Estimates of overall intensity were influenced by changes in the concentration of the odorant $[F(3,33)=$ $18.69, p<.01]$ and tastant $[F(3,33)=29.05, p<.01]$, but not by the interaction of the odorant and the tastant. A comparison of the arithmetic estimates of overall intensity of the nine combinations of the odorant/tastant mixtures with the sum of the arithmetic estimates of the unmixed components of the odor of ethyl butyrate and the taste of sucrose indicated that the estimates of overall intensity were only $70 \%$ as great as the sum of the estimates of smell and taste.

In Table 2, the estimates subjects gave to the smell of ethyl butyrate are compared with the estimates given to the overall intensity of the combination of the smell of ethyl butyrate and the taste of water. As can be seen in this table, average estimates of overall intensity were less than the estimates of the smell of ethyl butyrate. In Table 3, the estimates subjects gave to the taste of sucrose are compared with the estimates subjects gave to the overall intensity of the combination of the taste of sucrose and the smell of water vapor. Again, estimates of overall intensity were less than the estimates of the taste of sucrose.

Because, as discussed above, the calculations of $k_{s}$ and $k_{t}$ can be influenced by the presence of either retronasal smell or referred taste, we conducted a third experiment to determine how the taste of sucrose was affected by a sniff of ethyl butyrate and how the smell of ethyl butyrate was affected by a taste of sucrose.

\section{EXPERIMENT 3}

\section{Method}

Twenty-four undergraduate students, experimentally naive as to the psychophysical techniques of scaling smell and taste, were asked to estimate the lengths of lines, the intensity of the smell of distilled water and three concentrations of ethyl butyrate, and the taste of water and three concentrations of sucrose (three repetitions for each smell and taste; the concentrations were the same as those used in Experiment 2). The method and order of presentation of the stimuli were identical to that described for Experiment 2. These smell and taste trials constituted the initial battery of tests and were completed by all 24 subjects.
Table 2

Comparisons of Perceived Smell and Overall Intensity of Ethyl Butyrate

\begin{tabular}{lccr}
\hline & \multicolumn{3}{c}{ Log Percent Odorant Concentration } \\
\cline { 2 - 4 } & -2.00 & -1.40 & -0.80 \\
\hline $\begin{array}{l}\text { Smell } \\
\begin{array}{l}\text { Overall Intensity } \\
\text { (with water in } \\
\text { in the mouth) }\end{array}\end{array}$ & 5.12 & 7.43 & 9.79 \\
\hline
\end{tabular}

Table 3

Comparisons of Perceived Taste and Overall Intensity of Sucrose

\begin{tabular}{|c|c|c|c|}
\hline & \multicolumn{3}{|c|}{ Log Percent Tastant Concentration } \\
\hline & 0.70 & 1.00 & 1.30 \\
\hline Taste & 3.91 & 6.40 & 10.31 \\
\hline $\begin{array}{l}\text { Overall Intensity } \\
\text { (with water vapor } \\
\text { in the nose) }\end{array}$ & 2.90 & 6.44 & 7.61 \\
\hline
\end{tabular}

Following this initial battery of tests, 12 of the subjects were asked to estimate the intensity of the taste of distilled water and the three concentrations of sucrose with vapor of distilled water or one of the three concentrations of ethyl butyrate in the nose. The subjects were instructed to smell, then taste, then smell the solution again before answering the question "How intense does the solution taste?" All combinations of the four odorants and four tastants were presented twice, for a total of 32 trials.

Following the initial battery of tests (described above), the remaining 12 subjects were asked to estimate the intensity of the smell of distilled water and the three concentrations of ethyl butyrate with distilled water or one of the three concentrations of sucrose in the mouth. The subjects were instructed to taste, then smell, the solution before answering the question "How intense does the solution smell?" Intensity estimates were made while the tastant was in the subject's mouth. All combinations of the four odorants and four tastants were presented twice, for a total of 32 trials.

\section{Results}

For each subject, the arithmetic mean of the estimates of the odor and taste of each concentration was calculated. Estimates given by 1 subject to the smell of ethyl butyrate and the taste of sucrose were greater than 3.0 standard deviations from the mean; as a result, the data from this subject were not included in the analysis of the data.

Intensity estimates of the taste of distilled water were significantly higher when it was paired with the smell of ethyl butyrate than when it was paired with the smell of distilled water $[F(3,30)=3.30, p<.05]$. Furthermore, when the smell of the highest concentration of ethyl butyrate $(0.16 \%)$ was paired with the taste of sucrose, estimates of the intensity of taste were significantly higher $(81 \%)$ than the estimates given when the smell of distilled water was paired with the taste of sucrose $[F(3,30)=$ $4.22, p<.05]$. Table 4 shows the effect of ethyl butyrate vapors in the nose on the taste of distilled water and the three concentrations of sucrose.

Because the smell of ethyl butyrate affected the taste of distilled water, the taste of distilled water could not be assumed to represent the zero-taste condition. Thus, 
Table 4

Effects of Ethyl Butyrate Vapor in the Nose on the Taste of Water or Sucrose Solutions

\begin{tabular}{lcccr}
\hline \multirow{2}{*}{$\begin{array}{c}\text { Sucrose } \\
\text { Concentrations }\end{array}$} & \multicolumn{4}{c}{ Ethyl Butyrate Concentrations } \\
\cline { 2 - 5 } & Water & Low & Medium & High \\
\hline Water & 0.68 & 1.11 & 1.33 & 1.40 \\
Low & 2.94 & 3.73 & 3.63 & 3.63 \\
Medium & 4.23 & 6.67 & 5.12 & 10.25 \\
High & 6.02 & 7.94 & 9.15 & 10.00 \\
\hline
\end{tabular}

Table 5

Effects of Sucrose in the Mouth on the Smell of Water or Ethyl Butyrate Solutions

\begin{tabular}{lrrrr}
\hline \multirow{2}{*}{$\begin{array}{l}\text { Ethyl Butyrate } \\
\text { Concentrations }\end{array}$} & Water & Low & Medium & High \\
\cline { 2 - 5 } \multicolumn{1}{c}{ Water } & 0.61 & 1.26 & 1.81 & 1.61 \\
Low & 4.54 & 6.71 & 5.60 & 7.44 \\
Medium & 7.01 & 7.32 & 9.52 & 9.25 \\
High & 10.16 & 11.69 & 11.79 & 13.10 \\
\hline
\end{tabular}

Table 6

Estimates of Overall Intensity for all Combinations of the Smell of Ethyl Butyrate and the Taste of Sucrose

\begin{tabular}{lrrrrrrr}
\hline & \multicolumn{4}{c}{ Smell Concentrations } \\
\cline { 2 - 4 } \cline { 6 - 7 } Taste & \multicolumn{3}{c}{ Actual* } & & \multicolumn{3}{c}{ Predicted $\dagger$} \\
\cline { 2 - 5 } \cline { 5 - 7 } Concentrations & Low & Medium & High & & Low & Medium & High \\
\hline Low & 6.14 & 6.29 & 8.68 & & 6.00 & 7.25 & 8.53 \\
Medium & 8.59 & 8.83 & 12.21 & & 8.07 & 9.32 & 10.60 \\
High & 12.64 & 13.12 & 14.64 & & 11.32 & 12.57 & 13.85 \\
\hline
\end{tabular}

*On the average, estimates of overall intensity given by the subjects were $70 \%$ as large as the sum of the estimates for taste and smell. †On the average, estimates of the overall intensity predicted by the model were $68 \%$ as large as the sum of the estimates for taste and smell.

the values given to the taste of distilled water when the vapor of ethyl butyrate was in the nose (Table 4) were added to the values given to the smell of ethyl butyrate (Table 2) when calculating $k_{s}$. That is, $k_{s}=$ overall intensity/(smell + referred taste with water in the mouth). Using these numbers, estimates of overall intensity were $54 \%$ as large, on the average, as estimates of smell and referred taste (i.e., $k_{s}=0.54$ ).

Intensity estimates of the smell of distilled water did not differ significantly whether it was paired with the taste of sucrose or the taste of distilled water (Table 5). Although sucrose apparently does not produce the sensation of a smell, estimates of the intensity of the smell of ethyl butyrate were higher (31\%) when it was paired with the taste of the $10 \%$ or $20 \%$ sucrose solution than when it was paired with the taste of distilled water $[F(3,33)=$ $5.05, p<.01]$.

Because the referred smell from sucrose in the mouth did not significantly affect the smell of distilled water, $k_{t}=$ (overall intensity with water vapor in the nose)/(taste of sucrose). That is, it seems reasonable to assume that the smell of water approximates the zero-smell condition. On the average, estimates of overall intensity were $83 \%$ as large as the estimates of taste (i.e., $k_{t}=0.83$ ).
Using these calculated values of $k_{s}$ and $k_{t}$, and the estimates subjects gave to the smell of ethyl butyrate and the taste of sucrose, calculations of overall intensity were derived for each of the nine odorant/tastant combinations (Table 6). The model predicted estimates of overall intensity to be $68 \%$ as great as the sum of the estimates of smell and taste. Thus, the data given by the subjects (70\%) and the data predicted by the model are in good agreement.

\section{CONCLUSIONS}

Considering the differences in the timing of the delivery of the olfactory and gustatory components, and the differences created by presenting the olfactory stimulus to the external nares or the mouth, the results obtained with the open-cup delivery system (Experiment 1) and the Two-Module Delivery System (Experiment 2) are remarkably similar. That is, as the results of Experiment 1 demonstrate, when the scaling techniques of Enns and Hornung (1985) are used with the odorant placed in the mouth (open-cup delivery system), the overall intensity of a flavor stimulus is less than the sum of the intensities of taste and smell. This was also observed in Experiment 2, when the odorant was placed in front of the external nares (Two-Module Delivery System). Thus, the method of stimulus delivery does not seem to be responsible for the differences beteween the observations of Enns and Hornung (1985) and the data from studies that suggest that the intensity of flavor is equal to the sum of the intensities of taste and smell (Murphy \& Cain, 1980; Murphy et al., 1977).

One of the important findings of the present study is that the kinds of judgments (overall intensity or modalityspecific) can significantly affect the perception of intensity (Table 2). It is interesting to note that there is a reduction in the perceived overall intensity, compared with perceived intensity of taste alone, when only water vapor is in the nose. Likewise, there is a reduction in the perceived overall intensity, compared to perceived intensity of smell alone, when water is present in the mouth. These reductions occur even though there is no apparent opportunity for cross-modal suppression, masking, or interference. These observations support the involvement of a more central (cognitive) mechanism.

In the studies of Murphy and Cain (1980) and Murphy et al. (1977), complete additivity was obtained when estimates of the overall intensity of the odorant/tastant mixtures were compared with the sum of the estimates of the overall intensity of the odorant and the overall intensity of the tastant. A similar analysis of the data in the present study gives an equivalent answer. That is, when the sums of the estimates of the overall intensity of the smell (Table 2) and the overall intensity of the taste (Table 3) are compared with the estimates of overall intensity of the mixtures (Table 6), the additivity is $97 \%$.

Thus, the differences between the studies that show complete additivity and those that report a suppression seem not to be due to the delivery system. Rather, these 
differences seem to be due to the way in which the intensities of the odorants and tastants were determined. That is, if subjects are asked to rate the overall intensity of a smell or taste, they will, on the average, give a rating lower than they would give to the intensity of the taste or smell itself. The explanation for this phenomenon is not yet known. Certainly it is difficult to imagine that this is a peripheral phenomenon. Thus, parsimony suggests that the explanation involves a more central (cognitive) mechanism.

However, the present paper goes beyond this discussion to propose a mathematical model to describe the relationship between smell, taste, and overall intensity. The model, with its constants, has implications beyond a description of the additivity itself. The determination of the values of the constants might shed some light on the relationship between retronasal and nasal olfaction, and perhaps might even suggest a mechanism of the process of referred taste. It might be of further value in suggesting which compounds are likely to demonstrate referred taste.

For the model described above, the summations of smell and taste, each reduced by a constant factor $\left(k_{s}\right.$ and $k_{t}$, respectively), predicted accurately the estimates of overall intensity. Ideally, the values for the constant factors should be determined experimentally from the ratio of the estimates of overall intensity to the estimates of intensity of smell or taste when stimulation by the other modality is equal to zero. The present results indicate, however, that while the subjects were sniffing ethyl butyrate, the taste was not equal to zero. Thus, this referred taste effect had to be included in the calculation of $k_{s}$.

The fact that the model is so accurate at predicting estimates of overall intensity with the inclusion of the estimates of referred taste within the $k$ term for smell suggests one of two possibilities. First, perhaps the referred taste intensity ratings are so small as to not significantly influence the scaling of overall intensity. Second, perhaps the slope of the psychophysical function for the intensity of referred taste is close (or equal) to the slope of the psychophysical function for the intensity of the smell of ethyl butyrate. Since the data in Table 4 suggest that the first explanation is not the case, the second seems to be the most reasonable. Thus, the $k_{s}$ term is apparently an average of the effects on overall intensity of the smell and referred taste of ethyl butyrate.

It should prove interesting to compare the slopes of refered taste or retronasal smell to taste and smell psychophysical functions derived when the tastant and odorant are at low concentrations. If the slopes of the smell and referred taste curves were not approximately equal, the model would always predict intensity ratings which at some concentrations were higher than those observed and at other concentrations were lower than those observed. If referred taste could be shown to represent a psychophysical function similar to that seen with smell, then perhaps referred taste could be considered to be a central (cognitive) confusion. However, the answer to this and other questions relating to referred taste and retronasal smell remain to be answered.

When $k_{s}$ and $k_{\mathrm{t}}$ are not equal, the values for the percent suppression across all stimulus pairs should not be exactly equal. The left panel of Table 7 contains the actual mean values for all stimulus pairs for the magnitude estimate for overall intensity divided by the sum of the estimates of smell and taste (for the remainder of this paper this expression will be termed "the amount of reduction"). As expected, the data show that when the taste is low and the smell is high (top right point in this panel), the amount of reduction reflects mostly the influence of $k_{s}(.54)$. However, when taste is high and smell is low (lower left point), the amount of reduction reflects more the influence of $k_{t}(.84)$. Note also that the values along the diagonal representing low/low to high/high smell/taste combinations are intermediate to the values of $k_{s}$ and $k_{t}$, reflecting approximately equal contributions of smell and taste to overall intensity (and the amount of reduction is an approximate average of $k_{s}$ and $k_{t}$ ).

The right panel of Table 7 contains the amount of reduction predicted by the model. Note that besides predicting the average across all concentrations, the model is able to also predict the high, low, and intermediate points in the matrix. This seems to give further support to the predictive value of the model.

Obviously, the model does not predict exactly the amount of reduction seen at all points. To some extent this deviation represents the variability of the technique of absolute magnitude estimation. However, on the basis of a point-by-point analysis, it would seem that the current estimate for $k_{\mathrm{t}}$ is perhaps a bit low. (Note that the model predicts a somewhat lower amount of reduction when taste is high.) This is not surprising, inasmuch as the calculated $k_{t}$ showed a considerable amount of variability across all concentrations (Table 3 ). Perhaps if the sample size were increased, the variability around $k_{\mathrm{t}}$ would be reduced and so an even better estimate for this value could be obtained. It seems reasonable to suggest that in this case the value of $k_{t}$ might be somewhat higher than the value reported here.

In one sense, the discussion of the intensity of flavor, with its two definitions of olfaction, mirrors what Rozin (1982), in discussing the quality of flavor, described as

Table 7

Magnitude Estimates of Taste and Smell Divided by Overall Intensity for all Combinations of the Smell of Ethyl Butyrate and the Taste of Sucrose

\begin{tabular}{|c|c|c|c|c|c|c|}
\hline \multirow{3}{*}{$\begin{array}{c}\text { Taste } \\
\text { Concentrations }\end{array}$} & \multicolumn{6}{|c|}{ Smell Concentrations } \\
\hline & \multicolumn{3}{|c|}{ Actual*: } & \multicolumn{3}{|c|}{ Predicted } \\
\hline & Low & Medium & High & Low & Medium & High \\
\hline Low & .68 & .55 & .63 & .66 & .64 & .62 \\
\hline Medium & .74 & .64 & .75 & .71 & .67 & .66 \\
\hline High & .81 & .74 & .74 & .73 & .71 & .69 \\
\hline
\end{tabular}

*On the average, estimates of overall intensity given by the subjects were $70 \%$ as large as the sum of the estimates for taste and smell. +On the average, estimates of the overall intensity predicted by the model were $68 \%$ as large as the sum of the estimates for taste and smell. 
the duality of the olfactory sense. However, when considering the overall intensity of the stimulus, we suggest that the duality of the olfactory sense reflects the manner in which olfaction is tested, rather than different functions of the olfactory system.

Certainly one lesson from the study of flavor is that the design of the experiment can itself influence the results and thus influence the conclusions about the functioning of a sensory system. Therefore we propose the above model in the hope that it will be a small addition to the discussion of how taste and smell affect the flavor of a chemosensory-stimulating compound.

\section{REFERENCES}

ANDERSON, N. H. (1970). Functional measurement and psychophysical judgment. Psychological Review, 77, 153-170.

Berglund, B., Berglund, U., Lindvall, T., Svensson, L. T. (1973). A quantitative principle of perceived intensity summation in odor mixtures. Journal of Experimental Psychology, 100, 29-38.

Burdach, K. J., Kroeze, J. H. A., \& Koster, E. P. (1984). Nasal, retronasal, and gustatory perception: An experimental comparison. Perception \& Psychophysics, 36, 205-208.

Cometro-Muniz, J. E. (1981). Odor, taste, and flavor perception of some flavoring agents. Chemical Senses, 6, 215-223.

EnNs, M. P., Hornung, D. E. (1985). Contributions of smell and taste to overall intensity. Chemical Senses, 10, 357-366.

Garcia-Medina, M. A. (1981). Flavor-odor taste interactions in solutions of acetic acid and coffee. Chemical Senses, 6, 13-22.

GillaN, D. G. (1983). Taste-taste, odor-odor, and taste-odor mixtures: Greater suppression within than between modalities. Perception \& Psychophysics, 33, 183-185.

HoRnung, D. E., ENNs, M. P. (1984). The independence and integration of olfaction and taste. Chemical Senses, 9, 97-106.

MURPHY, C., \& CAIN, W. S. (1980). Taste and olfaction: Independence vs. interaction. Physiology \& Behavior, 24, 601-605.

Murphy, C., Cain, W. S., Bartoshuk, L. M. (1977). Mutual action of taste and olfaction. Sensory processes, 1, 204-211.

RozIN, P. (1982). Taste-smell confusions and the duality of the olfactory sense. Perception \& Psychophysics, 31, 397-401.

ZwisloCKI, J. J., \& Goodman, D. A. (1980). Absolute scaling of sensory magnitudes: A validation. Perception \& Psychophysics, 28, 28-38.

(Manuscript received April 15, 1985; revision accepted for publication May 18, 1986.) 